\title{
Developing a Multimedia Product for the World Wide Web
}

\author{
Linda Lisle, Scott Isensee, and Jianming Dong \\ IBM Corp. \\ 11400 Burnet Road \\ Austin, TX 78758
}

\section{INTRODUCTION}

Our group is responsible for developing the IBM HCI and Ease of Use web sites. The goals in developing these sites have included investigating and demonstrating appropriate uses of multimedia in web design. Through our experience developing these sites, research we have conducted, user feedback we have received, literature reviews, interviews with web site developers, and related activities, we have learned a number of valuable lessons. This paper summarizes the most important of those lessons.

\section{SYNCHRONIZATION OF MEDIA}

Synchronization of media is particularly difficult on the Web. Issues include speed of the processor on the client, speed of the internet connection, and load on the server. These unknowns make it difficult to select media attributes such as frame rates for video or animation and sampling rates for audio quality. Multimedia is not yet a standard element of Web browsers. It typically requires a plug-in.

We decided to provide our data in multiple formats. We used animation technologies such as Flash or Shockwave which could be used by those with fast connections and provided downloadable versions such as Java applications for users with slower connections. The illustration below shows an example of several frames from a Flash demo which gives a preview of an application which can be downloaded.
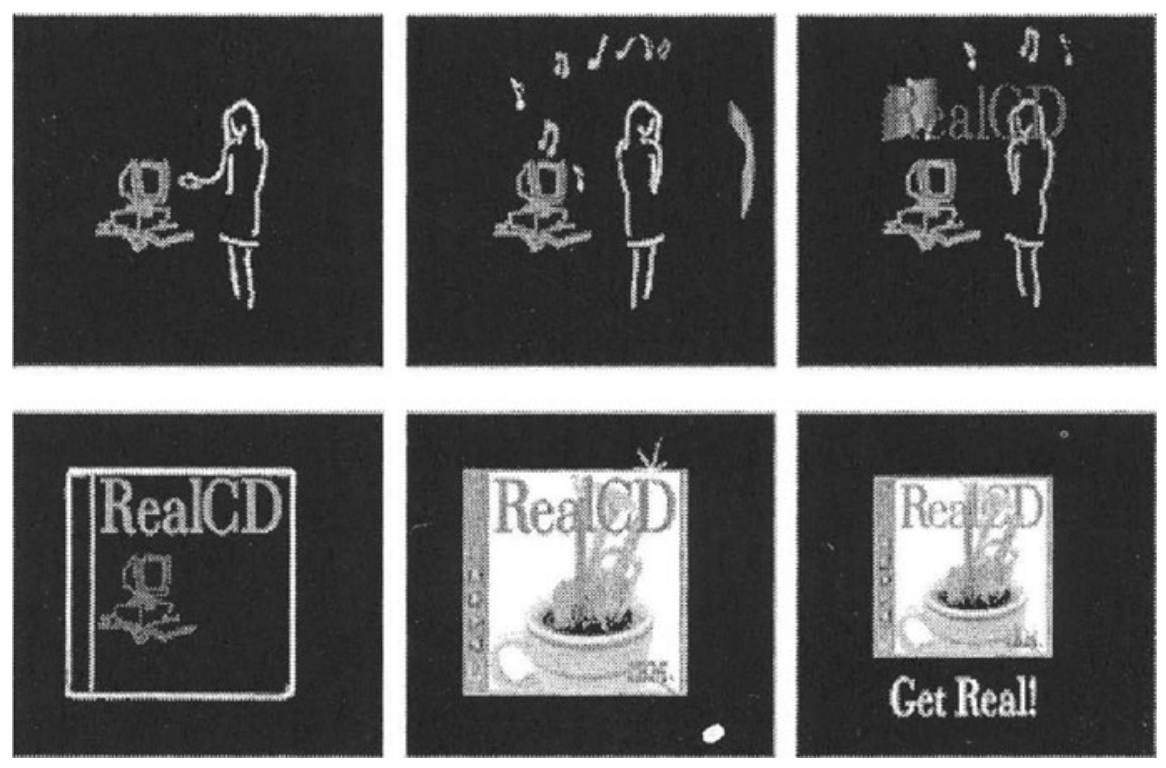

Figure 1. Animation 


\section{DO'S AND DON'TS}

Through experience, testing, and user feedback, we have learned a great deal about what works well and what doesn't in user interface design for the web. To institutionalize this knowledge for ourselves and others, we created a set of Web Guidelines which are available at http://www.ibm.com/easy/

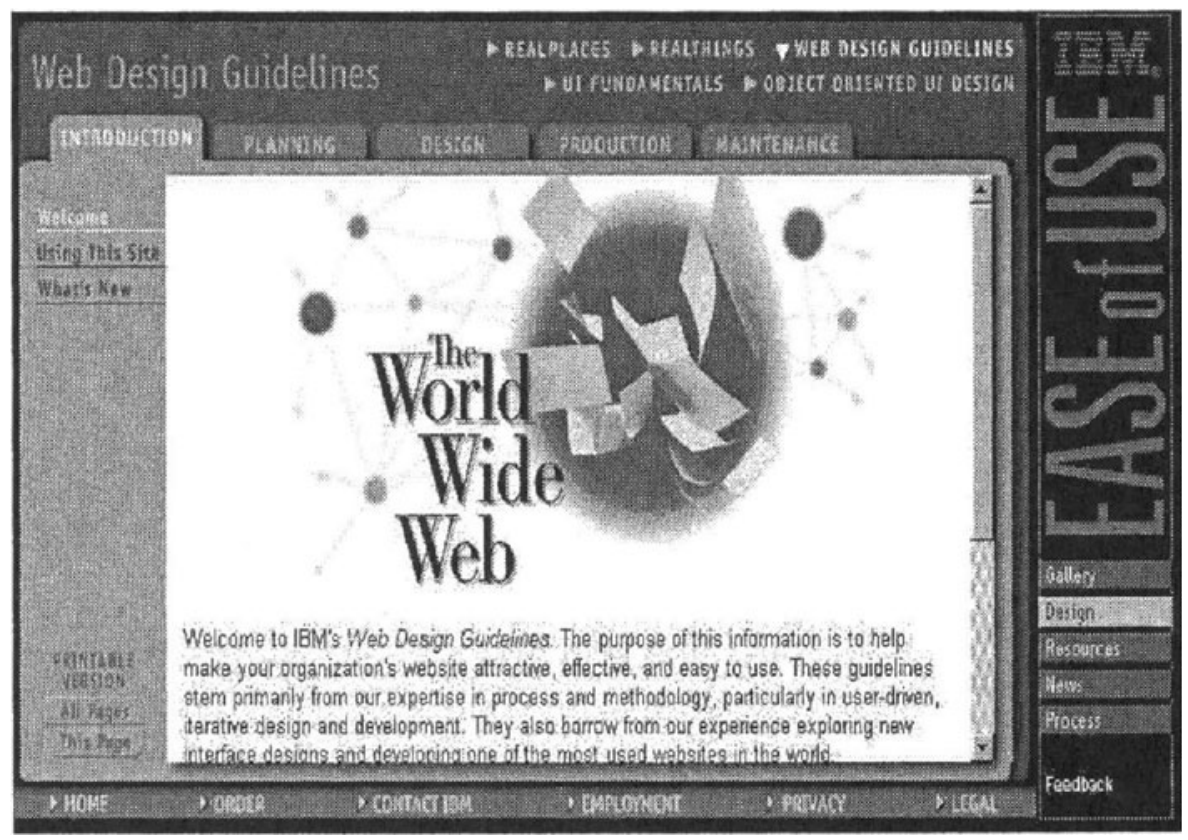

Figure 2. Web guidelines

\section{PROJECT MANAGEMENT}

The difficulty of developing for the Web is increasing as the available media and technologies increase. Multimedia development requires people with greater ranges of skills: visual design, audio design, programming, etc. The activities of these people must be coordinated. Classical project management techniques such as Gant charts have proven very helpful. The Web provides a great communication vehicle for the project members. We coordinate our team through project management charts on our intranet site.

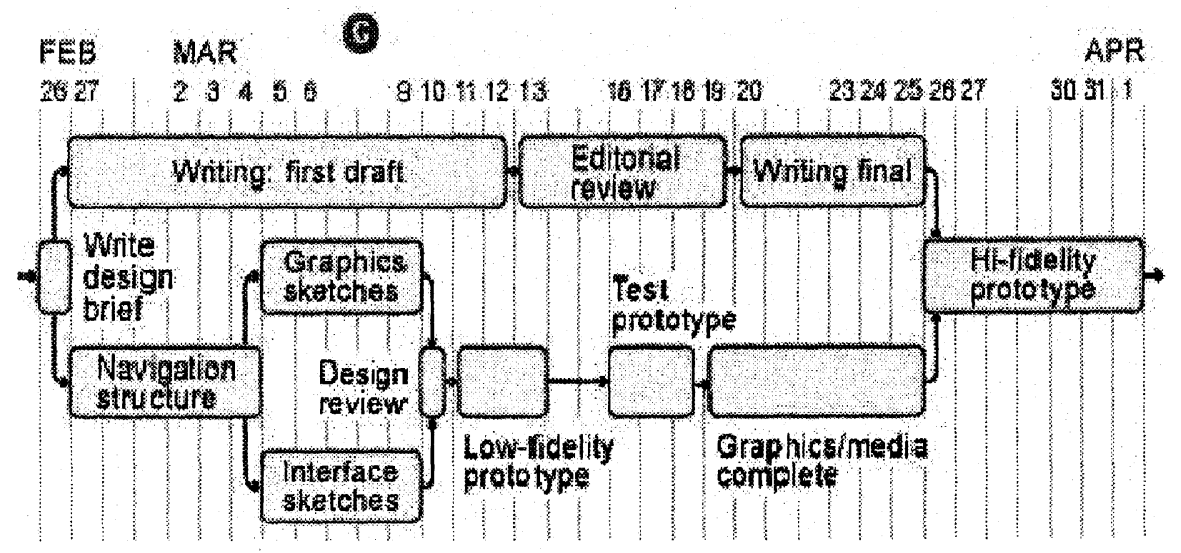

Figure 3. Gant chart 
The web provides a hyperlinked information structure which users must navigate through. In our testing we found users would become "lost in hyperspace" jumping from link to link. We were able to change this experience from one of going from place to place to one where information is brought to the user in a single place. We accomplished this by using frames to reserve parts of the page for navigation and refreshing only the content frame as the user navigated throughout the site. The figure shows an example of a page layout which provides navigation panes that remain constant while a content pane is refreshed.

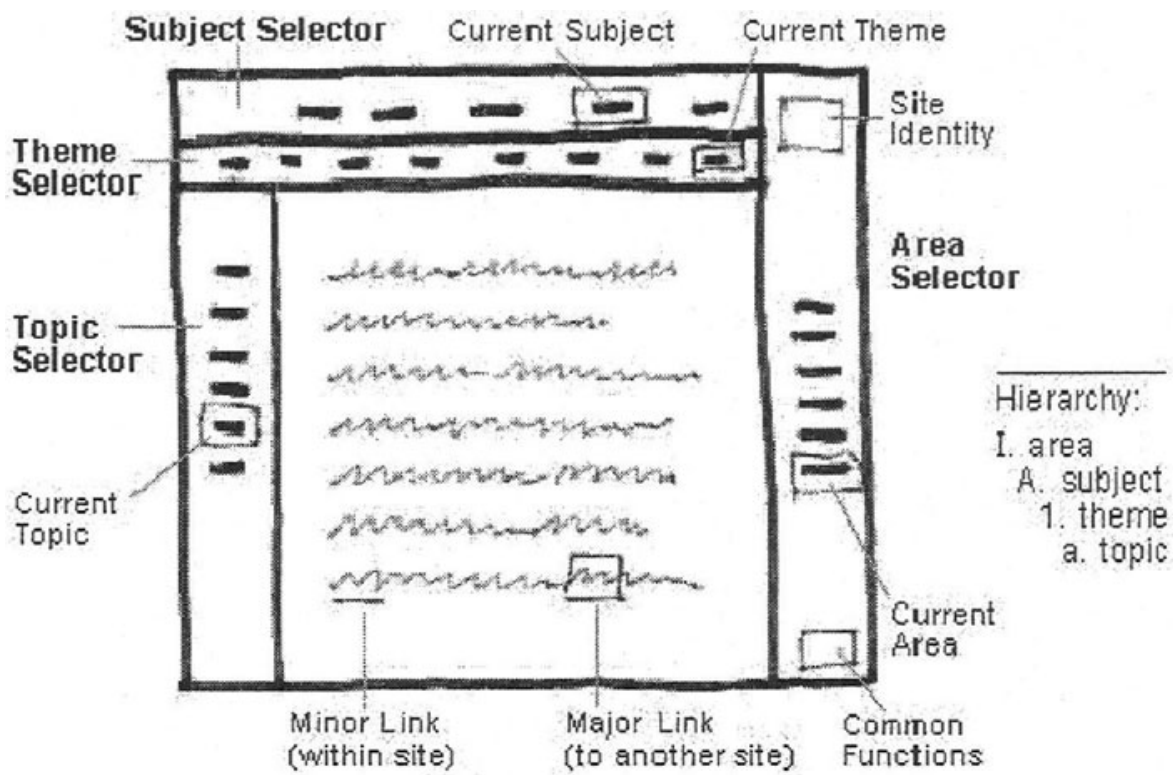

Figure 4. Page layout

\section{RISING LEVEL OF EXPECTATION}

Users are coming to expect increasingly higher levels of quality in the media they encounter on the web. If the quality of presentation is not sufficient, you can't capture the readers interest long enough to deliver your message. We have addressed this by employing professional visual designers and by developing leading edge visual and audio elements in cooperation with our research division.

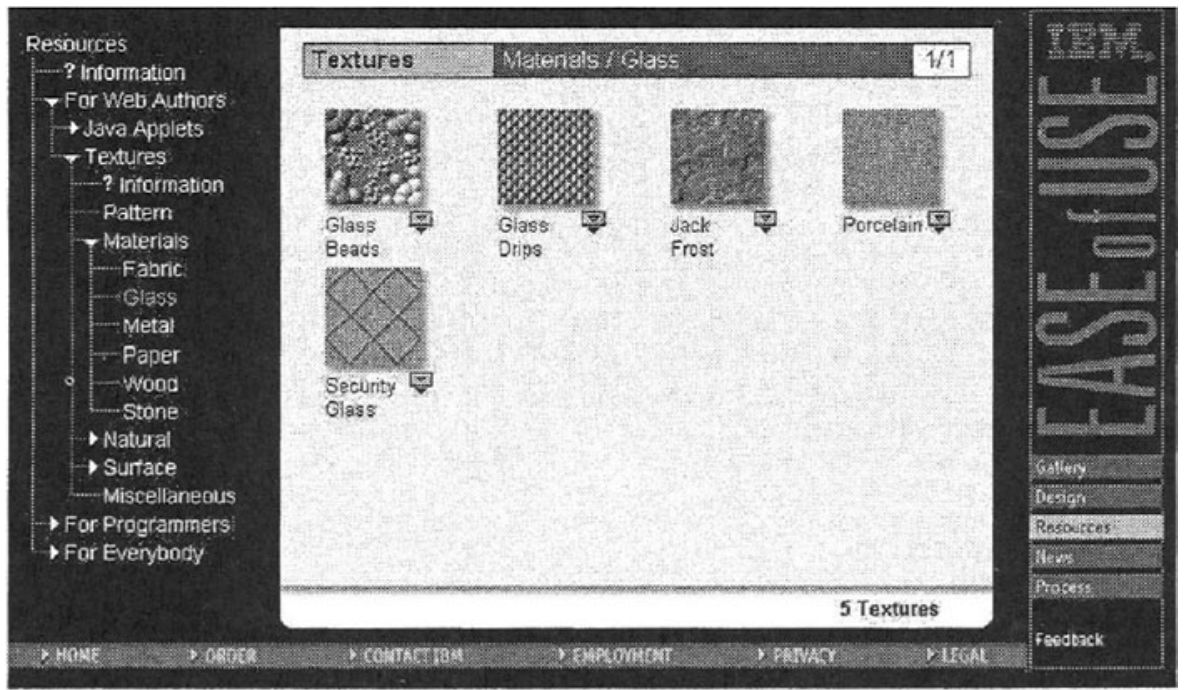

Figure 5. Visual elements 